
\title{
25 Research Soure \\ Simulated Ventilation of Two Patients With a Single Ventilator in a Pandemic Setting
}

Pascal Schepat

Anesthesiology, Intensive Care, Pain Therapy and Emergency Medicine

Benjamin Kober

Anetshesiology, Intensive Care Medicine, Pain Therapy and Emergency Medicine

Martin Eble

Anesthesiolgy, Intensive Care Medicine, Pain Therapy and Emergency Medicine

Volker Wenzel

Anesthesiology, Intensive Care Medicine, Pain Therapy and Emergency Medicine

Holger Herff ( $\square$ holger.herff@uk-koeln.de)

Department of Anesthesiology, PAN Clinic https://orcid.org/0000-0002-7380-4013

\section{Research Article}

Keywords: 3D Printing, ARDS, COVID-19 pandemic, double patient ventilation, flow limitation, ventilator

Posted Date: November 9th, 2021

DOI: https://doi.org/10.21203/rs.3.rs-146213/v2

License: (c) (1) This work is licensed under a Creative Commons Attribution 4.0 International License.

Read Full License 


\section{Abstract}

Background: Simultaneous ventilation of two patients, e.g., due to a shortage of ventilators in a pandemic, may result in hypoventilation in one patient and hyperinflation in the other patient.

Methods: In a simulation of double patient ventilation using artificial lungs with equal compliances $\left(70 \mathrm{~mL} \cdot \mathrm{mbar}^{-1}\right)$, we tried to voluntarily direct gas flow to one patient by using 3D-printed y-adapters and stenosis adapters during volume-, and pressure-controlled ventilation. Subsequently, we modified the model using a special one-way valve on the limited flow side and measured in pressure-controlled ventilation with the flow sensor adjusted on either side in a second and third setup. In the last setup, we also measured with different lung compliances.

Results: Volume- or pressure-controlled ventilation using standard connection tubes with the same compliance in each lung resulted in comparable minute volumes in both lungs, even if one side was obstructed to $3 \mathrm{~mm}(6.6 \pm 0.2 \mathrm{vs} .6 .5 \pm 0.1 \mathrm{~L}$ for volume-controlled ventilation, $p=.25$ continuous severe alarm and 7.4 $\pm 0.1 \mathrm{vs} .6 .1 \pm 0.1 \mathrm{~L}$ for pressure-controlled ventilation, $p=.02$ no alarm). In the second setup, pressurecontrolled ventilation resulted at a $3 \mathrm{~mm}$ flow limitation in minute ventilation of $9.4 \pm 0.3 \mathrm{vs} 3.5 \pm 0.1 \mathrm{~L} \cdot \mathrm{min}^{-1}$, $\mathrm{p}=.001$. In a third setup using the special one-way valve and the flow sensor on the unobstructed side, pressure-controlled ventilation resulted at a $3 \mathrm{~mm}$ flow limitation in minute ventilation of $7.4 \pm 0.2 \mathrm{vs} 3 \pm 0 \mathrm{~L} \cdot \mathrm{min}^{-1}$, at the compliance of $70 \mathrm{~mL} \cdot \mathrm{mbar}^{-1}$ for both lungs, $7.2 \pm 0 \mathrm{vs} 4.1 \pm 0 \mathrm{~L} \cdot \mathrm{min}^{-1}$, at the compliances of $50 \mathrm{vs} .70 \mathrm{~mL} \cdot \mathrm{mbar}^{-1}$, and $7.2 \pm 0.2 \mathrm{vs} 5.7 \pm 0 \mathrm{~L} \cdot \mathrm{min}^{-1}$, at the compliance of $30 \mathrm{vs}$. $70 \mathrm{~mL} \cdot \mathrm{mbar}^{-1}$ (all $\left.\mathrm{p}=.001\right)$.

Conclusions: Overriding a modern intensive care ventilator's safety features are possible, thereby ventilating two lungs with one ventilator simultaneously in a laboratory simulation using 3D-printed yadapters. Directing tidal volumes in different pulmonary conditions towards one lung using 3D-printed flow limiters with diameters $<6 \mathrm{~mm}$ was also possible. While this ventilation setting was technically feasible in a bench model, it would be volatile, if not dangerous in a clinical situation.

\section{Background}

The COVID-19 pandemic revealed in hospitals a dramatic shortages of experienced staff, medical equipment, and especially a lack of ventilators. Even in the fall of 2021 after nearly two years of the pandemic there is an actual crisis in ICU resources due to COVID-19 in Romania and Bulgaria leading to emergency transfer of patients to other EU countries. Such situations were unimaginable in western industrialised countries before the COVID-19 Pandemic. In desperate situations in the last two years, it was even attempted to share one ventilator for two patients. However, due to different resistance and lung compliance, this may result in hypoventilation in one patient and hyperinflation in the other patient, which may cause lung damage[1], and adversely affect morbidity and mortality. 
A simple solution to distribute the tidal volume of one ventilator between two patients may be a 3D printed y-adapter, which has been reported before during the 9/11 terrorist attacks and Hurricane Katrina in New Orleans[2]. Recent reports from the United States show that comparable devices have been used in the Covid-19 Pandemic as well[3]. Historical studies demonstrated this strategy in a laboratory setting ${ }^{[2]}$. However, as the tidal volume cannot be distributed adequately to each patient mainly due to different resistance and lung compliance, this may result in hypoventilation in one patient, and hyperinflation in the other patient. This may result in hypercarbia or even hypoxemia in a given patient with the "worse" lung, while the better lung may be hyperinflated and thus become severely damaged. To avoid such a misdistribution between patients, we developed a 3D printed stenosis adapter that can be inserted in the respirator tube of one patient. Thus, in theory, the tidal volume can be distributed variably between patients.

We evaluated gas distribution in a ventilation bench model using artificial lungs with equal lung compliance during volume-controlled and pressure-controlled ventilation by inserting stenosis adapters of 2-9mm, respectively. Subsequently, we modified the model using a special one-way valve and evaluated in pressure-controlled ventilation mode the influence of these stenosis adapters on gas distribution between lungs with different lung compliance settings representing ventilation of standard intensive care patients vs. ARDS patients, e.g., due to Covid-19. Our formal hypothesis was that there would be no difference between groups.

\section{Materials And Methods Ethics approval}

This study is a completely technical simulation with no participants. Thus, no ethical approval was required.

\section{Experimental setup}

We developed a y-adapter fitting to a modern ventilation device according to ISO 5356-1 (Figure 1A). This adapter can be used both in the in- and the expiratory branch of the ventilation tubes. Accordingly, we developed flow limitation devices with a diameter of $2-9 \mathrm{~mm}$ that could be inserted in the ventilator tubes (Figure 1B).

Both pieces consisted of carbon fibre (Multec Carbon, Multec, Illmensee, Germany), and were printed on a 3D printer (Multec Multirap M800, Multec, Illmensee, Germany). The average printing time was 4 hrs; the adapter could be disinfected and sterilised. For lung and airway simulation, we used a double test lung with lung compliance and airway resistance being adjustable in each test lung separately (dual Adult TTL $\circledast$ - Model 5600i, Michigan Instruments, Grand Rapids, MI). Thus, each side of the lung was set to represent the airway characteristics of an individual patient; in result, the dual lung represented the airway 
characteristics of two patients. For determining minute ventilation, we included a respirometer Ferraris, Hertford, UK) in the tubes connecting the ventilator to each of the separated lungs simulating one patient.

We adjusted the Y-adapter with a modern ventilator (Hamilton C6, Bonaduz, Switzerland) in different positions, to ventilate both test lungs independently (Figure 2).

In the first setup, we used $\mathrm{Y}$-adapters in both the in- and expiratory circuits that we connected with standard double-lumen ventilation tubes to test lungs. The flow limiter was adjusted on side of lung $B$ directly behind the inspiratory $y$-adapter, the flow sensor was set on the circuit to test lung $A$. This setting enabled expiratory gas to flow back from both test lungs to the ventilator. (Figure $2 \mathrm{~A}$ ). In the second setup, we used only one $y$-adapter in the inspiratory circuit and connected only lung $B$ via a standard double-lumen connection tube, allowing expiratory gas to flow back to the ventilator only from lung $B$.

Test lung A was connected to the ventilator with a single lumen connection tube; at the distal end prior to the test lung $A$ we fixed a connection piece of an AMBU Mark $V$ bag-valve device (Ambu Glostrup, Copenhagen, Denmark); thus, the expiratory gas was directly vented into the ambient air near the test lung $A$ and did not flow back to the ventilator via a double-lumen tube as in setup one. The flow limiter was also integrated in circuit $B$ again as in setup one. The flow sensor was adjusted to the double lumen side of circuit B (Figure 2B). In a third setup, we connected lung A to a standard double-lumen connection tube, allowing expiratory gas to flow from the lung $A$ back to the ventilator. The one-way connection tube was connected to lung B; we adjusted both the flow limiter distally of the $Y$-adapter and the expiratory valve of an AMBU Mark V bag valve device (Ambu Glostrup, Copenhagen, Denmark) in circuit B. In the result, ventilation of lung $B$ was both limited and expiratory gas flow from lung $B$ was directed into the ambient air not flowing back to the ventilator. The flow sensor was adjusted to the standard double lumen side of the circuit A (Figure 2C).

\section{Experimental procedure:}

In total, six experiments were performed in the afore mentioned setups; each experiment was performed for $1 \mathrm{~min}$ and repeated six times. The first two experiments were done in setup one. In the first experiment (Figure 2A), both lungs were ventilated in volume-controlled mode with a compliance of $30 \mathrm{~mL} \bullet \mathrm{mbar}^{-1}$ simulating moderate ARDS in both lungs (respiratory rate $12 \cdot \mathrm{min}^{-1}$, tidal volume $1200 \mathrm{~mL}, \mathrm{p}_{\max } 20 \mathrm{mbar}$, PEEP $5 \mathrm{mbar} \mathrm{FiO}_{2}$ 0.21, I:E 1:2). The first measurement was without a flow limiter. Subsequently, flow limiters with decreasing diameters $(9-2 \mathrm{~mm})$ were integrated into the inspiratory tube connected to lung $B$. The second experiment was done in pressure-controlled mode in the same first setup (respiratory rate 12• $\mathrm{min}^{-1}, \mathrm{P}_{\max } 20 \mathrm{mbar}$, PEEP 5mbar, $\mathrm{FiO}_{2}$ 0.21, I:E 1:2). In all pressure-controlled experiments, the peak pressure was adjusted to achieve a tidal volume of $600 \mathrm{~mL}$ in the unobstructed lung $A$. Since the measurements were done with different compliances, this was adjusted for each experiment.

The third experiment was performed in the second setup (Figure 2B). The peak inspiratory pressure was chosen to achieve tidal volume in lung $A$ of $600 \mathrm{~mL}$. Compliance of test lung $A$ was $70 \mathrm{~mL} \cdot \mathrm{mbar}^{-1}$ 
simulating a healthy lung. The fourth experiment was pressure-controlled ventilation in analogy to experiments 2 and 3 in the third setup (Figure 2C) with a compliance of $70 \mathrm{~mL} \cdot \mathrm{mbar}^{-1}$ in both lungs. The fifth experiment was again done in setup three (Figure $2 \mathrm{C}$ ) and compared the ventilatory parameters of test lung $A$ with a compliance of $50 \mathrm{~mL} \cdot \mathrm{mbar}^{-1}$ with ventilatory parameters of test lung $B$ with $70 \mathrm{~mL} \cdot \mathrm{mbar}^{-1}$. The sixth experiment was also done in setup three (Figure $2 \mathrm{C}$ ) and compared the ventilatory parameters of test lung $A$ with a compliance of $30 \mathrm{~mL} \cdot \mathrm{mbar}^{-1}$ with ventilatory parameters of test lung $B$ with $70 \mathrm{~mL} \cdot \mathrm{mbar}^{-1}$, respectively. Pressure for ventilation was always adjusted to achieve tidal volumes of $600 \mathrm{~mL}$ in the unobstructed side.

\section{Statistical analysis:}

Statistical evaluation was performed with Sigmaplot 14 (Systat, San Jose, CA) using Student's $t$-test after Shapiro Wilk Analysis for normality and Equal Variance Test (Brown-Forsythe). If a normality test failed, the Mann-Whitney rank-sum test was applied. Data are given as mean \pm standard deviation. $P$ values $<0.05$ were considered significant.

\section{Results}

In experiments one and two, we measured comparable minute volumes in both lungs using either volume or flow-controlled ventilation regardless of the use of flow limiters (Tables 1 and 2). The volumecontrolled ventilation mode resulted in a severe continuous disconnection alarm, although ventilation was adequate (Table 1). In the third experiment, flow limitation distributed tidal volume to lung $A$. However, the lung A was moderately hyperventilated when flow limiters with smaller diameters were used (Table 3). In experiment, four flow limiters decreased volume on the obstructed side effectively, while the lung A was ventilated adequately (Table 4). In experiments five and six, simultaneous ventilation of two lungs with different compliances was possible by including flow limiters of $<6 \mathrm{~mm}$ flow diameter in the tube to lung B with better compliance (Tables 5 and 6). 
Table 1

Volume-controlled mode with the same compliance in both lungs of $30 \mathrm{ml} / \mathrm{mbar}$. Flow limitation on circuit $B$ flow sensor on circuit A, double hose circuit for lungs A and B.

\begin{tabular}{|c|c|c|c|c|c|}
\hline $\begin{array}{l}\text { Y-adapter } \\
\text { inside diameter }\end{array}$ & $\begin{array}{l}\text { Test lung } \\
\text { A } \\
\text { M.V. } \\
\text { (L/min) }\end{array}$ & $\begin{array}{l}\text { Test lung B } \\
\text { M.V. } \\
\text { (L/min) }\end{array}$ & $\begin{array}{l}\text { F.S. C6 (A) } \\
\text { M.V. } \\
\text { (L/min) }\end{array}$ & $\begin{array}{l}\text { Hamilton C6 } \\
\text { pPeak } \\
\text { (mbar) }\end{array}$ & $\begin{array}{l}\text { P } \\
\text { Test lung A vs. } \\
\text { B }\end{array}$ \\
\hline $\begin{array}{l}\text { A: } \varnothing 19 \mathrm{~mm}, \mathrm{~B}: \varnothing \\
19 \mathrm{~mm}\end{array}$ & $6.4 \pm 0.4$ & $6.8 \pm 0.3^{*}$ & $6.2 \pm 0.1$ & $28 \pm 0$ & 0.25 \\
\hline $\begin{array}{l}\text { A: } \varnothing 19 \mathrm{~mm}, \mathrm{~B}: \varnothing \\
9 \mathrm{~mm}\end{array}$ & $6.3 \pm 0.4$ & $6.9 \pm 0.3^{*}$ & $6,3 \pm 0.1$ & $28 \pm 0$ & 0.03 \\
\hline $\begin{array}{l}\text { A: } \varnothing 19 \mathrm{~mm}, \mathrm{~B}: \varnothing \\
8 \mathrm{~mm}\end{array}$ & $6.4 \pm 0.3$ & $6.7 \pm 0.1^{*}$ & $6.1 \pm 0.1$ & $28 \pm 0$ & 0.12 \\
\hline $\begin{array}{l}\text { A: } \varnothing 19 \mathrm{~mm}, \mathrm{~B}: \varnothing \\
7 \mathrm{~mm}\end{array}$ & $6.6 \pm 0.3$ & $6.7 \pm 0.2^{*}$ & $5.8 \pm 0.1$ & $28 \pm 0$ & 0.09 \\
\hline $\begin{array}{l}\text { A: } \varnothing 19 \mathrm{~mm}, \mathrm{~B}: \varnothing \\
6 \mathrm{~mm}\end{array}$ & $6.6 \pm 0.4$ & $6.7 \pm 0.2^{*}$ & $6.1 \pm 0.1$ & $28 \pm 0$ & 0.12 \\
\hline $\begin{array}{l}\text { A: } \varnothing 19 \mathrm{~mm}, \mathrm{~B}: \varnothing \\
5 \mathrm{~mm}\end{array}$ & $6.7 \pm 0.2$ & $6.6 \pm 0.1^{*}$ & $6.0 \pm 0.2$ & $28 \pm 0$ & 0.35 \\
\hline $\begin{array}{l}\text { A: } \varnothing 19 \mathrm{~mm}, \mathrm{~B}: \varnothing \\
4 \mathrm{~mm}\end{array}$ & $6.5 \pm 0.2$ & $6.5 \pm 0.1^{*}$ & $5.8 \pm 0.1$ & $27 \pm 0$ & 0.35 \\
\hline $\begin{array}{l}\text { A: } \varnothing 19 \mathrm{~mm}, \mathrm{~B}: \varnothing \\
3 \mathrm{~mm}\end{array}$ & $6.5 \pm 0.2$ & $6.4 \pm 0.1^{*}$ & $5.7 \pm 0.2$ & $27 \pm 0$ & 0.92 \\
\hline $\begin{array}{l}\text { A: } \varnothing 19 \mathrm{~mm}, \mathrm{~B}: \varnothing \\
2 \mathrm{~mm}\end{array}$ & $6.6 \pm 0.2$ & $6.5 \pm 0.1^{*}$ & $5.7 \pm 0.1$ & $27 \pm 0$ & 0.22 \\
\hline \multicolumn{6}{|c|}{$\begin{array}{l}A=\text { Inside diameter of the } y \text {-adapter according to ISO } 5356-1 ; B=\text { With and without }(19 \mathrm{~mm}) \text { limitation } \\
\text { of the inside diameter of the flow limiter; M.V.; minute ventilation; * }{ }^{*} \text { Critical alert: Disconnection on } \\
\text { patient-side alarm. If flow sensor is connected prior to test lung B and not prior the y-adapter stop of } \\
\text { ventilation; F.S. = flow sensor on circuit A }\end{array}$} \\
\hline
\end{tabular}


Table 2

Pressure-controlled ventilation with the same compliance of $30 \mathrm{~mL} / \mathrm{mbar}$ in both lungs. Flow limitation, and flow sensor in front of $y$-adapter, double hose circuit for lungs $A$ and $B$.

\begin{tabular}{|c|c|c|c|c|c|}
\hline $\begin{array}{l}\text { Y-adapter } \\
\text { inside diameter }\end{array}$ & $\begin{array}{l}\text { Test lung } \\
\text { A } \\
\text { M.V. } \\
\text { (L/min) }\end{array}$ & $\begin{array}{l}\text { Test lung B } \\
\text { M.V. } \\
\text { (L/min) }\end{array}$ & $\begin{array}{l}\text { F.S. on A } \\
\text { M.V. } \\
\text { (L/min) }\end{array}$ & $\begin{array}{l}\text { Hamilton C6 } \\
\text { pPeak } \\
\text { (mbar) }\end{array}$ & $\begin{array}{l}\text { P } \\
\text { Test lung A vs. } \\
\text { B }\end{array}$ \\
\hline $\begin{array}{l}\text { A: } \varnothing 19 \mathrm{~mm}, \mathrm{~B}: \varnothing \\
19 \mathrm{~mm}\end{array}$ & $7.6 \pm 0.2$ & $7.8 \pm 0.3$ & $7.1 \pm 0.1^{\star}$ & $28 \pm 0$ * & 0.34 \\
\hline $\begin{array}{l}\text { A: } \varnothing 19 \mathrm{~mm}, \mathrm{~B}: \varnothing \\
9 \mathrm{~mm}\end{array}$ & $7.4 \pm 0.2$ & $7.9 \pm 0.2$ & $6.8 \pm 0.1^{*}$ & $28 \pm 0$ * & 0.03 \\
\hline $\begin{array}{l}\text { A: } \varnothing 19 \mathrm{~mm}, \mathrm{~B}: \varnothing \\
8 \mathrm{~mm}\end{array}$ & $7.5 \pm 0.3$ & $7.9 \pm 0.2$ & $7.3 \pm 0.1^{*}$ & $28 \pm 0$ * & 0.08 \\
\hline $\begin{array}{l}\text { A: } \varnothing 19 \mathrm{~mm}, \mathrm{~B}: \varnothing \\
7 \mathrm{~mm}\end{array}$ & $7.5 \pm 0.1$ & $8.0 \pm 0.1$ & $7.2 \pm 0.1^{*}$ & $28 \pm 0$ * & 0.03 \\
\hline $\begin{array}{l}\text { A: } \varnothing 19 \mathrm{~mm}, \mathrm{~B}: \varnothing \\
6 \mathrm{~mm}\end{array}$ & $7.5 \pm 0.1$ & $7.9 \pm 0.1$ & $7.1 \pm 0.1^{*}$ & $28 \pm 0 *$ & 0.03 \\
\hline $\begin{array}{l}\text { A: } \varnothing 19 \mathrm{~mm}, \mathrm{~B}: \varnothing \\
5 \mathrm{~mm}\end{array}$ & $7.4 \pm 01$ & $7.5 \pm 0.1$ & $7.1 \pm 0.1^{*}$ & $28 \pm 0 *$ & 0.04 \\
\hline $\begin{array}{l}\text { A: } \varnothing 19 \mathrm{~mm}, \mathrm{~B}: \varnothing \\
4 \mathrm{~mm}\end{array}$ & $7.4 \pm 0.1$ & $7.4 \pm 0.1$ & $7.1 \pm 0.1^{*}$ & $26 \pm 0$ * & 0.35 \\
\hline $\begin{array}{l}\text { A: } \varnothing 19 \mathrm{~mm}, \mathrm{~B}: \varnothing \\
3 \mathrm{~mm}\end{array}$ & $7.4 \pm 0.1$ & $7.2 \pm 0.1$ & $7.0 \pm 0.1^{*}$ & $26 \pm 0$ * & 0.03 \\
\hline $\begin{array}{l}\text { A: } \varnothing 19 \mathrm{~mm}, \mathrm{~B}: \varnothing \\
2 \mathrm{~mm}\end{array}$ & $7.4 \pm 0.1$ & $7.1 \pm 0.1$ & $7.1 \pm 0.1^{*}$ & $26 \pm 0$ * & 0.03 \\
\hline
\end{tabular}


Table 3

Pressure-controlled ventilation with the same compliance in both lungs of $70 \mathrm{~mL} / \mathrm{mbar}$. Flow limitation on circuit B and flow sensor on circuit B. Single hose in circuit A and a double hose in circuit B.

\begin{tabular}{|c|c|c|c|c|c|}
\hline $\begin{array}{l}\text { Y-adapter } \\
\text { inside diameter }\end{array}$ & $\begin{array}{l}\text { Test lung } \\
\text { A } \\
\text { M.V. } \\
\text { (L/min) }\end{array}$ & $\begin{array}{l}\text { Test lung B } \\
\text { M.V. } \\
\text { (L/min) }\end{array}$ & $\begin{array}{l}\text { Hamilton } \\
\text { C6 } \\
\text { M.V. } \\
\text { (L/min) }\end{array}$ & $\begin{array}{l}\text { Hamilton C6 } \\
\text { pPeak } \\
\text { (mbar)\# }\end{array}$ & $\begin{array}{l}\text { P for M.V. } \\
\text { Test lung A vs. } \\
\text { B }\end{array}$ \\
\hline $\begin{array}{l}\text { A: } \varnothing 19 \mathrm{~mm}, \mathrm{~B}: \varnothing \\
19 \mathrm{~mm}\end{array}$ & $8.1 \pm 0.4$ & $7.8 \pm 0.4$ & $6.8 \pm 0.2$ & $14 \pm 0$ & 0.3 \\
\hline $\begin{array}{l}\text { A: } \varnothing 19 \mathrm{~mm}, \mathrm{~B}: \varnothing \\
9 \mathrm{~mm}\end{array}$ & $8.9 \pm 0.2$ & $7.9 \pm 0.5$ & $6.9 \pm 0.11$ & $14 \pm 0$ & 0.04 \\
\hline $\begin{array}{l}\text { A: } \varnothing 19 \mathrm{~mm}, \mathrm{~B}: \varnothing \\
8 \mathrm{~mm}\end{array}$ & $9.3 \pm 0.3$ & $8.0 \pm 0.4$ & $6.6 \pm 0.2$ & $14 \pm 0$ & 0.001 \\
\hline $\begin{array}{l}\text { A: } \varnothing 19 \mathrm{~mm}, \mathrm{~B}: \varnothing \\
7 \mathrm{~mm}\end{array}$ & $9.4 \pm 0.2$ & $7.8 \pm 0.1$ & $6.2 \pm 0.2$ & $14 \pm 0$ & 0.002 \\
\hline $\begin{array}{l}\text { A: } \varnothing 19 \mathrm{~mm}, \mathrm{~B}: \varnothing \\
6 \mathrm{~mm}\end{array}$ & $9.6 \pm 0.1$ & $7.9 \pm 0.3$ & $6.7 \pm 0$ & $14 \pm 0$ & 0.002 \\
\hline $\begin{array}{l}\text { A: } \varnothing 19 \mathrm{~mm}, \mathrm{~B}: \varnothing \\
5 \mathrm{~mm}\end{array}$ & $10.5 \pm 0$ & $7.9 \pm 0.1$ & $6.9 \pm 0.1$ & $14 \pm 0$ & 0.002 \\
\hline $\begin{array}{l}\text { A: } \varnothing 19 \mathrm{~mm}, \mathrm{~B}: \varnothing \\
4 \mathrm{~mm}\end{array}$ & $10.2 \pm 0.1$ & $6 \pm 0.1$ & $5.4 \pm 0.1$ & $14 \pm 0$ & 0.001 \\
\hline $\begin{array}{l}\text { A: } \varnothing 19 \mathrm{~mm}, \mathrm{~B}: \varnothing \\
3 \mathrm{~mm}\end{array}$ & $9.4 \pm 0.3$ & $3.5 \pm 0.1$ & $3.0 \pm 0.2$ & $13 \pm 0$ & 0.001 \\
\hline $\begin{array}{l}\text { A: } \varnothing 19 \mathrm{~mm}, \mathrm{~B}: \varnothing \\
2 \mathrm{~mm}\end{array}$ & \multicolumn{5}{|l|}{ Failed } \\
\hline \multicolumn{6}{|c|}{$\begin{array}{l}A=\text { Inside diameter of the } y \text {-adapter according to ISO } 5356-1 ; B=\text { With and without }(19 \mathrm{~mm}) \text { limitation } \\
\text { of the inside diameter by the flow limiter; * M.V.; minute ventilation; No machine-side error messages if } \\
\text { flow sensor is attached to test lung B. \# Adjustment of the upper ventilation pressure to } 14 \text { mbar due } \\
\text { to a change in lung compliance test lung A }\end{array}$} \\
\hline
\end{tabular}


Table 4

Pressure-controlled ventilation with the same compliance in both lungs of $70 \mathrm{ml} / \mathrm{mbar}$. Flow limitation on circuit B and flow sensor on circuit A. Single hose in circuit A and a double hose in circuit B.

\begin{tabular}{|c|c|c|c|c|c|}
\hline \multirow{2}{*}{$\begin{array}{l}\text { Y-adapter } \\
\text { inside diameter }\end{array}$} & $\begin{array}{l}\text { Test lung } \\
\text { A }\end{array}$ & $\begin{array}{l}\text { Test lung } \\
\text { B }\end{array}$ & $\begin{array}{l}\text { Hamilton } \\
\text { C6 }\end{array}$ & \multirow{2}{*}{$\begin{array}{l}\text { Hamilton C6 } \\
\text { pPeak } \\
\text { (mbar)\# }\end{array}$} & \multirow{2}{*}{$\begin{array}{l}\text { P } \\
\text { Test lung A vs. } \\
\text { B }\end{array}$} \\
\hline & $\begin{array}{l}\text { M.V. } \\
\text { (I/min) }\end{array}$ & $\begin{array}{l}\text { M.V. } \\
\text { (I/min) }\end{array}$ & $\begin{array}{l}\text { M.V. } \\
\text { (I/min) }\end{array}$ & & \\
\hline $\begin{array}{l}\text { A: } \varnothing 19 \mathrm{~mm}, \mathrm{~B}: \varnothing \\
19 \mathrm{~mm}\end{array}$ & $8.0 \pm 0.4$ & $7.8 \pm 0.4$ & $7.2 \pm 0,1$ & $14 \pm 0$ & 0.3 \\
\hline $\begin{array}{l}\text { A: } \varnothing 19 \mathrm{~mm}, \mathrm{~B}: \varnothing \\
9 \mathrm{~mm}\end{array}$ & $6.7 \pm 0.1$ & $6.6 \pm 0.1$ & $6.7 \pm 0,1$ & $14 \pm 0$ & 0.25 \\
\hline $\begin{array}{l}\text { A: } \varnothing 19 \mathrm{~mm}, \mathrm{~B}: \varnothing \\
8 \mathrm{~mm}\end{array}$ & $7.5 \pm 0.3$ & $6.5 \pm 0.2$ & $6.7 \pm 0$ & $14 \pm 0$ & 0.001 \\
\hline $\begin{array}{l}\text { A: } \varnothing 19 \mathrm{~mm}, \mathrm{~B}: \varnothing \\
7 \mathrm{~mm}\end{array}$ & $7.9 \pm 0$ & $7.0 \pm 0$ & $6.7 \pm 0$ & $14 \pm 0$ & 0.001 \\
\hline $\begin{array}{l}\text { A: } \varnothing 19 \mathrm{~mm}, \mathrm{~B}: \varnothing \\
6 \mathrm{~mm}\end{array}$ & $7.5 \pm 0.1$ & $6.5 \pm 0.1$ & $6.7 \pm 0$ & $14 \pm 0$ & 0.001 \\
\hline $\begin{array}{l}\text { A: } \varnothing 19 \mathrm{~mm}, \mathrm{~B}: \varnothing \\
5 \mathrm{~mm}\end{array}$ & $7.8 \pm 0.1$ & $6.5 \pm 0.1$ & $6.7 \pm 0$ & $14 \pm 0$ & 0.001 \\
\hline $\begin{array}{l}\text { A: } \varnothing 19 \mathrm{~mm}, \mathrm{~B}: \varnothing \\
4 \mathrm{~mm}\end{array}$ & $7.5 \pm 0$ & $5 \pm 0$ & $6.7 \pm 0$ & $14 \pm 0$ & 0.001 \\
\hline $\begin{array}{l}\text { A: } \varnothing 19 \mathrm{~mm}, \mathrm{~B}: \varnothing \\
3 \mathrm{~mm}\end{array}$ & $7.7 \pm 0.1$ & $3 \pm 0$ & $6.7 \pm 0$ & $14 \pm 0$ & 0.001 \\
\hline $\begin{array}{l}\text { A: } \varnothing 19 \mathrm{~mm}, \mathrm{~B}: \varnothing \\
2 \mathrm{~mm}\end{array}$ & $7.4 \pm 0.2$ & $0.8 \pm 0$ & $6.7 \pm 0$ & $14 \pm 0$ & 0.001 \\
\hline $\begin{array}{l}\text { A = Inside diamet } \\
\text { of the inside diam } \\
\text { flow sensor is att } \\
\text { to a change in lur }\end{array}$ & $\begin{array}{l}\text { y-adapte } \\
\text { the flow li } \\
\text { test lung } \\
\text { liance tes }\end{array}$ & $\begin{array}{l}\text { sording to } \\
\text { r; }{ }^{*} \text { M.V.; } \\
\text { :Adjustm } \\
\text { g A }\end{array}$ & $\begin{array}{l}356-1 ; \\
\text { ventila } \\
\text { the upp }\end{array}$ & $\begin{array}{l}\text { ith and without } \\
\text { No machine-sic } \\
\text { ntilation press }\end{array}$ & $\begin{array}{l}9 \mathrm{~mm} \text { ) limitation } \\
\text { aror messages if } \\
\text { to } 14 \text { mbar due }\end{array}$ \\
\hline
\end{tabular}


Table 5

Pressure-controlled ventilation with compliance of $50 \mathrm{~mL} / \mathrm{mbar}$ in Lung $\mathrm{A}$ and $70 \mathrm{ml} / \mathrm{mbar}$ in Lung B. Flow limitation on circuit B and flow sensor on circuit A. Single hose in circuit B and a double hose in circuit A.

\begin{tabular}{|c|c|c|c|c|c|}
\hline \multirow[t]{2}{*}{$\begin{array}{l}\text { Y-adapter } \\
\text { inside diameter }\end{array}$} & $\begin{array}{l}\text { Test lung } \\
\text { A }\end{array}$ & $\begin{array}{l}\text { Test lung } \\
\text { B }\end{array}$ & $\begin{array}{l}\text { Hamilton } \\
\mathrm{C6}\end{array}$ & \multirow{2}{*}{$\begin{array}{l}\text { Hamilton C6 } \\
\text { pPeak } \\
\text { (mbar)\# }\end{array}$} & \multirow{2}{*}{$\begin{array}{l}\text { P } \\
\text { Test lung A vs. } \\
\text { B }\end{array}$} \\
\hline & $\begin{array}{l}\text { M.V. } \\
\text { (I/min) }\end{array}$ & $\begin{array}{l}\text { M.V. } \\
(1 / \mathrm{min})\end{array}$ & $\begin{array}{l}\text { M.V. } \\
(1 / \mathrm{min})\end{array}$ & & \\
\hline $\begin{array}{l}\text { A: } \varnothing 19 \mathrm{~mm}, \mathrm{~B}: \varnothing \\
19 \mathrm{~mm}\end{array}$ & $7.4 \pm 0.1$ & $9.6 \pm 0.2$ & $7.2 \pm 0.1$ & $18 \pm 0$ & 0.001 \\
\hline $\begin{array}{l}\text { A: } \varnothing 19 \mathrm{~mm}, \mathrm{~B}: \varnothing \\
6 \mathrm{~mm}\end{array}$ & $7.6 \pm 0.2$ & $9.4 \pm 0.4$ & $6.9 \pm 0$ & $18 \pm 0$ & 0.001 \\
\hline $\begin{array}{l}\text { A: } \varnothing 19 \mathrm{~mm}, \mathrm{~B}: \varnothing \\
5 \mathrm{~mm}\end{array}$ & $7.6 \pm 0$ & $8.5 \pm 0.1$ & $6.9 \pm 0$ & $18 \pm 0$ & 0.001 \\
\hline $\begin{array}{l}\text { A: } \varnothing 19 \mathrm{~mm}, \mathrm{~B}: \varnothing \\
4 \mathrm{~mm}\end{array}$ & $7.4 \pm 0$ & $6.6 \pm 0$ & $6.9 \pm 0$ & $18 \pm 0$ & 0.001 \\
\hline $\begin{array}{l}\text { A: } \varnothing 19 \mathrm{~mm}, \mathrm{~B}: \varnothing \\
3 \mathrm{~mm}\end{array}$ & $7.2 \pm 0$ & $4.1 \pm 0$ & $6.9 \pm 0$ & $18 \pm 0$ & 0.001 \\
\hline $\begin{array}{l}\text { A: } \varnothing 19 \mathrm{~mm}, \mathrm{~B}: \varnothing \\
2 \mathrm{~mm}\end{array}$ & $7.2 \pm 0$ & $1.2 \pm 0.1$ & $6.9 \pm 0$ & $18 \pm 0$ & 0.001 \\
\hline
\end{tabular}


Table 6

Pressure-controlled ventilation with compliance of $30 \mathrm{~mL} / \mathrm{mbar}$ in Lung A and $70 \mathrm{ml} / \mathrm{mbar}$ in Lung B. Flow limitation on circuit B and flow sensor on circuit A. Single hose in circuit A and a double hose in circuit B.

\begin{tabular}{|c|c|c|c|c|c|}
\hline $\begin{array}{l}\text { Y-adapter } \\
\text { inside diameter }\end{array}$ & $\begin{array}{l}\text { Test lung } \\
\text { A } \\
\text { M.V. } \\
\text { (L/min) }\end{array}$ & $\begin{array}{l}\text { Test lung B } \\
\text { M.V. } \\
\text { (L/min) }\end{array}$ & $\begin{array}{l}\text { Hamilton } \\
\text { C6 } \\
\text { M.V. } \\
\text { (L/min) }\end{array}$ & $\begin{array}{l}\text { Hamilton C6 } \\
\text { pPeak } \\
\text { (mbar)\# }\end{array}$ & $\begin{array}{l}\text { P } \\
\text { Test lung A vs. } \\
\text { B }\end{array}$ \\
\hline $\begin{array}{l}\text { A: } \varnothing 19 \mathrm{~mm}, \mathrm{~B}: \varnothing \\
19 \mathrm{~mm}\end{array}$ & $7.0 \pm 0.1$ & $14.5 \pm 0.2$ & $7.2 \pm 0.1$ & $26 \pm 0$ & 0.001 \\
\hline $\begin{array}{l}\text { A: } \varnothing 19 \mathrm{~mm}, \mathrm{~B}: \varnothing \\
6 \mathrm{~mm}\end{array}$ & $7.5 \pm 0.1$ & $14.5 \pm 0.4$ & $6.7 \pm 0$ & $26 \pm 0$ & 0.001 \\
\hline $\begin{array}{l}\text { A: } \varnothing 19 \mathrm{~mm}, \mathrm{~B}: \varnothing \\
5 \mathrm{~mm}\end{array}$ & $7.4 \pm 0$ & $12 \pm 0.1$ & $6.7 \pm 0$ & $26 \pm 0$ & 0.001 \\
\hline $\begin{array}{l}\text { A: } \varnothing 19 \mathrm{~mm}, \mathrm{~B}: \varnothing \\
4 \mathrm{~mm}\end{array}$ & $7.3 \pm 0$ & $9 \pm 0$ & $6.7 \pm 0$ & $26 \pm 0$ & 0.001 \\
\hline $\begin{array}{l}\text { A: } \varnothing 19 \mathrm{~mm}, \mathrm{~B}: \varnothing \\
3 \mathrm{~mm}\end{array}$ & $7.2 \pm 0.2$ & $5.7 \pm 0$ & $6.7 \pm 0$ & $26 \pm 0$ & 0.001 \\
\hline $\begin{array}{l}\text { A: } \varnothing 19 \mathrm{~mm}, \mathrm{~B}: \varnothing \\
2 \mathrm{~mm}\end{array}$ & $7.2 \pm 0.2$ & $2.0 \pm 0.1$ & $6.7 \pm 0$ & $26 \pm 0$ & 0.001 \\
\hline \multicolumn{6}{|c|}{$\begin{array}{l}A=\text { Inside diameter of the } y \text {-adapter according to ISO } 5356-1 ; B=\text { With and without }(19 \mathrm{~mm}) \text { limitation } \\
\text { of the inside diameter by the flow limiter; }\end{array}$} \\
\hline
\end{tabular}

\section{Discussion}

In this study, we show the evaluation process of using flow limiters to direct tidal volume to one lung. Simulated ventilation of two patients with a single ventilator was technically possible employing both volume- and pressure-controlled ventilation when patient parameters were comparable. The preset lung's compliance did not impact results when it was identical in both lungs: The ventilator was powerful enough to ventilate both lungs with compliances as low as $30 \mathrm{~mL} / \mathrm{mbar}$, nor did it oversteer when both lungs were set to $70 \mathrm{~mL} / \mathrm{mbar}$. In a recently published study, the authors described that changes in airway mechanics in one patient might have less dangerous effects on the second patient when pressurecontrolled ventilation was used for double patient ventilation instead of volume-controlled ventilation[4]. We could not validate this effect since volume-controlled ventilation in our model was hampered by continuous disconnection alarms by the ventilator and ventilation was stopped by the ventilator [4]. Thus, we omitted the volume-controlled ventilation design and concentrated on pressure-controlled ventilation.

The limitation of gas flow by flow restrictors was not possible in setup one if standard double hose circuits were used on both lungs. We speculate that due to using two y-adapters in the in- and expiratory 
circuit, we elevated the pressure first in the unobstructed inspiratory circuit, and then in the expiratory circuit on this side. This may have resulted due to the second y-adapter on the expiratory side of the ventilator with a faster pressure elevation on the expiratory side of the obstructed circuit than on the inspiratory side. As a result, the obstructed lung may have been ventilated in a retrograde manner via the expiratory hose on the obstructed side. This is in accordance with the supplemental video, where delayed ventilation of the obstructed side was visible (Supplementary Video Files 1 and 2). In result, we were not able to direct gas to one lung using flow limiters in setup one.

We changed the setup by inducing a one-way valve with an additional PEEP adapter to lung A. As a result, only gas from lung $B$ streamed back to the ventilator, avoiding this error in setup two. When the ventilator's flow sensor was connected to the obstructed side, ventilation with efforts of the ventilator was still possible up to a diameter of $3 \mathrm{~mm}$, and it only failed at a diameter of $2 \mathrm{~mm}$. We suggest that modern, powerful ventilators can ventilate even through a stenosis of $3 \mathrm{~mm}$, being comparable to small neonate ventilation tubes, and thus overcome our volume limitation attempts on one side. However, it resulted in a hyperventilation of the non-obstructed side, which could be detrimental in a real clinical setting. Thus, we omitted this setting as well. We then further modified to setup three which worked reliably: Lung A was ventilated in a conventional setting using a standard double-lumen hose and the flow sensor was fixed there, too; the inspiratory lumen of a bypass to lung B was obstructed by a flow limiter and a one-way valve expired ventilation gas into the ambient air on side $B$. With lung compliance of $70 \mathrm{~mL} / \mathrm{mbar}$ on both sides, it was possible to variably decrease minute ventilation with our flow limiter on the obstructed side to even $0.8 \mathrm{~L} / \mathrm{min}$. Furthermore, ventilation was possible even in very different lung compliance settings if the worse lung $A$ was ventilated in a conventional setting, whereas a bypass to the healthy lung $B$ was obstructed (experiments five and six). The intention of this study was always measured in different compliance settings. We deliberately did not start with different compliances since that is easier at the beginning in a proof of concept attempt. Since experiments 1-3 failed even with the same compliances, they were thus not pursued. We deliberately integrated the failing attempts 1-3 in this manuscript demonstrating the complexity of the whole developmental process. This study shows that using a specific ventilator in two patients without clearing it's specific functioning setup before in test lungs may result in immediate failure.

To significantly direct minute ventilation to one patient, in general, flow limiters of $<6 \mathrm{~mm}$ had to be used. This contrasts sharply with previous suggestions where far larger diameters $(11-16 \mathrm{~mm})$ were suggested for this purpose; using such large diameters in our setting resulted in severe hyperinflation and lung overinflation in the lungs with better compliances [5]. In other studies, adjustable flow limiters or a Hoffmann clamp adjusted to ventilation have been used to increase inspiratory resistance; however, the exact diameters are not known in these settings and cannot be standardised [6, 7]. Use of pressure control valves for the distribution of gas between two patients as a completely different solution has been reported before, too [8]. At the moment it is not known which system may be the most promising; our solution is standardised and completely reproducible in regard to used diameters. To further standardise the model, we deliberately decided to combine the flow sensor always with the standard double-lumen hose as the original manufacturer's product; the other side was always connected with the 
one-way valve. The lung on the one-way valve side can be ventilated with the PEEP level preset on the ventilator plus an additional PEEP by adjusting a simple PEEP valve (e.g. MARK V BVD, Ambu Glostrup, Copenhagen, Denmark) to the one-way valve. However, in our experiment the one-way valve was adjusted to the better side where the additional PEEP does clinically make not much sense.

Venting exhaled contaminated gas into ambient air poses a risk of infection in most intensive care ventilators, especially in our model this risk may be increased since patients would be lying near each other due to technical reasons. Thus, additional safety measures, such as special decontamination filters might be mandatory as described before; in addition, this might decrease the infection risk for medical staff, too.[6] Further additional one-way valves can be integrated in the circuits without much effort not allowing exhaled gas flowing from one patient to the other.

Interestingly, the ventilator is indeed complete blind to the real situation on the side without the flow sensor; e.g., it calculated a minute volume of $7 \mathrm{I}$ in experiment 6 , whereas we measured minute volumes > $\mathrm{20L} / \mathrm{min}$ in the test lungs. Thus, it may even not detect detrimental divergencies. Thus, overriding a modern ventilator's safety features would create a setting requiring very skilled monitoring by a highly experienced healthcare worker and would still be dangerous; the minimum personal monitoring parameters might be TV for each patient, maximum airway pressure and PEEP levels. However, the COVID-19 pandemic showed us that even health care systems in the highest developed countries might collapse, such as in Bergamo/Italy, Strasbourg/France, or New York City, where mechanical ventilation demand exceeded capacity by far. As a result, death tolls rose dramatically in these regions.[9] Case reports from the U.S. show that despite the concerns of the SCCM, comparable systems had been used at the height of the Pandemic in spring $2020[4,6,9]$. Governor Cuomo of New York explicitly authorised the use of such systems on March 26th, 2020[3]; we do not know how countries with less sophisticated intensive care medicine systems handle this challenge. Ventilating two patients with a single ventilator for bridging or not, it may still be a question in the actual third wave of the pandemic in many regions in the world that can be posed to many intensivists with only a few weeks of warning.

In a joint declaration, American and European societies for critical care medicine postulate not to ventilate two patients with a single ventilator[10]. Based on our measurements, we definitely support this recommendation - if the characteristics of respiratory system parameters change, this will result in hyperinflation of the better lungs and hypoventilation of the worse. Moreover, patients cannot be allowed to ventilate spontaneously when flow limiters are applied, as the patient on the limited side would be in a situation of acute airway obstruction, thus impairing any weaning attempts. Furthermore, no proper alarm management is possible if the ventilator's safety features have to be overridden before this strategy could work. Additionally, patients would have to be physically positioned next to each other, thus facilitating the exchange of aerosols and cross infections. Denying one patient a ventilator clearly impacts that patient, however, the use of the system described in two patients may result in injury or death in both. This remains an ethical dilemma that can only be answered in a situation of acute crisis. Accordingly, despite functioning perfectly well in this laboratory setting, such a setting is only imaginable in a desperate situation for a limited time after emergency intubation in deeply sedated patients for bridging until a 
second ventilator becomes available [11, 12]. Furthermore, our data - if so -supports only the use of the specific ventilator used in this study; using a different ventilator might need a substantially different concept. Without testing this specific concept before the attempt may result in immediate failure as our failed setups one and two showed us.

In addition, a self-printed adapter is not in accordance with the E.U. or any other guidelines for medical products. However, in this model our hypothesis could be examined without any risk to patients. We deliberately ventilated in this model both test lungs with $600 \mathrm{ml}$ each, a value at the upper scale and that only may be acceptable in large male adult patients. Although the ratio of gas distribution between patients should be unaffected in a system of linear lung compliance, smaller TVs on the unobstructed side may, despite misdistribution, have resulted in still acceptable TVs on the obstructed side. Thus, we choose a tidal volume at the upper potential scale for the unobstructed side to demonstrate how fast detrimental TVs might result in a lung with better compliance, if the flow is not limited adequately. Although it is the tidal volume that may impact a lung in a clinical setting, we indicated minute volume in this study since that was the value we measured in the test lungs. Measuring tidal volume manually in two lungs with each ventilation using mechanical flow meters is quite complicated, whereas it is technically easy and more exact to measure the minute volume after 60 seconds. Average tidal volume can be easily calculated by division. Furthermore, this laboratory model is even more precise than any human study or animal experiment. Thus, this model always poses a risk to detect statistically significant differences due to its exactness that is clinically merely not relevant. Last, all results cannot be extrapolated to other ventilators.

\section{Conclusions}

Overriding a modern intensive care ventilator's safety features is possible, thereby ventilating two lungs simultaneously with one ventilator in a laboratory simulation using 3D-printed y-adapters. Directing tidal volumes in different pulmonary conditions towards one lung using 3D-printed flow limiters with diameters $<6 \mathrm{~mm}$ was also possible. While this ventilation setting was technically feasible in a bench model, it would be volatile, if not dangerous in a clinical situation.

\section{Abbreviations}

ARDS - Adult respiratory distress syndrome

MV - Minute Volume

PEEP - Positive end-expiratory pressure

TV - Tidal Volume

\section{Declarations}




\section{Ethics approval}

Since this was a completely technical simulation with no participants, no ethical approval was required.

\section{Consent to participate}

Not applicable.

\section{Consent for publication}

Not applicable.

\section{Availability of data and materials}

All data generated used or analyzed during this study are included in the published article.

\section{Competing interests}

The authors declare that they do not have any conflict of interest.

\section{Funding}

This study was supported by institutional resources.

\section{Author contributions}

All authors contributed to the study conception. The first protocol was designed by Pascal Schepat, Volker Wenzel and Holger Herff. Material preparation, data collection and analysis were performed by Pascal Schepat, Benjamin Kober, Martin Eble and Holger Herff. The first draft of the manuscript was written by Pascal Schepat and Holger Herff and all authors commented on previous versions of the manuscript. All authors read and approved the final manuscript. This manuscript contains significant parts of the doctoral thesis of P. Schepat, MD.

\section{Acknowledgements}

We thank Tobias Schlegel, M.D. (General Hospital Böblingen, Germany) to design the initial blueprint of the $y$-adapter and the flow limiters. A special thanks go to Petra and Florian Rapp (Multec, Illmensee, Germany) to revise the designs, and print the adapters due to the COVID-19 Pandemic.

\section{Preprint}

This manuscript was published as preprint.[13]

\section{References}


1. Ranney ML, Griffeth V, Jha AK. Critical Supply Shortages - The Need for Ventilators and Personal Protective Equipment during the Covid-19 Pandemic. N Engl J Med 2020;382 18: e41.

2. Neyman G, Irvin CB. A single ventilator for multiple simulated patients to meet disaster surge. Acad Emerg Med 2006;13 11: 1246-9.

3. Allen J, Brown N. One ventilator, two patients: New York hospitals shift to crisis mode: Reuters. 2020.

4. Rodriguez-Villar S. Sharing a single ventilator ("In vitro"). Med Intensiva 2020.

5. Lai BK, Erian JL, Pew SH, Eckmann MS. Emergency Open-source Three-dimensional Printable Ventilator Circuit Splitter and Flow Regulator during the COVID-19 Pandemic. Anesthesiology 2020;133 1: 246-8.

6. Levin MA, Shah A, Shah R, Kane E, Zhou G, Eisenkraft JB et al. Differential Ventilation Using Flow Control Valves as a Potential Bridge to Full Ventilatory Support during the COVID-19 Crisis. Anesthesiology 2020;133 4: 892-904.

7. Clarke AL, Stephens AF, Liao S, Byrne TJ, Gregory SD. Coping with COVID-19: ventilator splitting with differential driving pressures using standard hospital equipment. Anaesthesia 2020;75 7: 872-80.

8. Herrmann J, Fonseca da Cruz A, Hawley ML, Branson RD, Kaczka DW. Shared Ventilation in the Era of COVID-19: A Theoretical Consideration of the Dangers and Potential Solutions. Respir Care 2020;65 7: 932-45.

9. Briegel J. Virusinfektion und fehlende Beatmungsgeräte - ein Déjà-vu? Anesthesist 2020;69: 314-5.

10. Consensus Statement on Multiple Patients Per Ventilator: Society of Critical Care Medicine. 2020.

11. Notz Q, Herrmann J, Stumpner J, Schmid B, Schlesinger T, Kredel M et al. [Anesthesia and intensive care ventilators: differences and usability in COVID-19 patients]. Anaesthesist 2020;69 5: 316-22.

12. Bohm SH, Kremeier P, Tusman G, Reuter DA, Pulletz S. [Volumetric capnography for analysis and optimization of ventilation and gas exchange]. Anaesthesist 2020;69 5: 361-70.

\section{Figures}

\section{Figure 1A.}

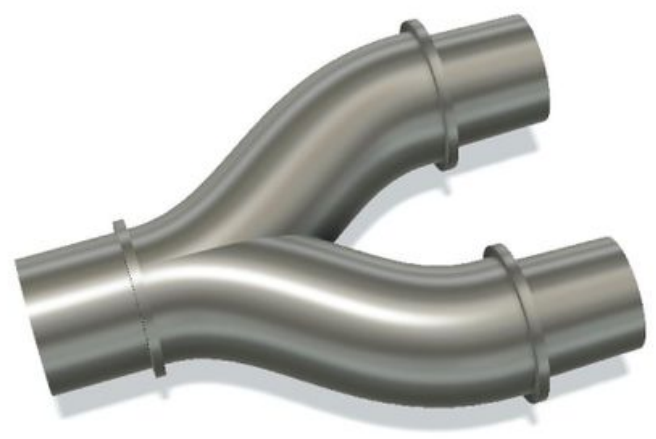

Figure 1B.
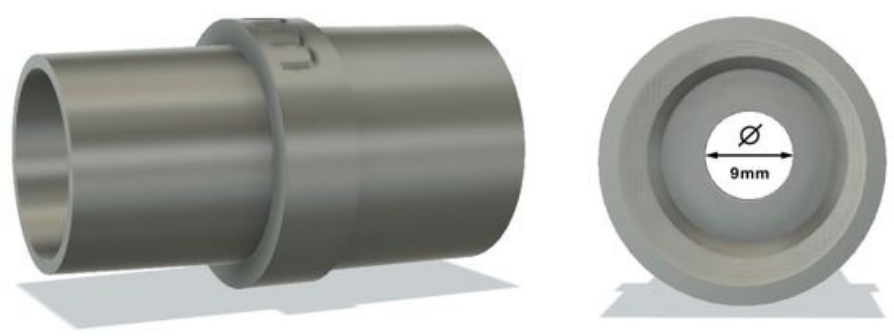
A Draft of a 3D printable y-adapter can be used to divert gas flow to two patients according to ISO 53561. B Draft of a 3D printable "Flow limiter "created in Autodesk Fusion 360 according to ISO 5356-1: variable inside diameter from $9-2 \mathrm{~mm}$.

Figure 2 .

A

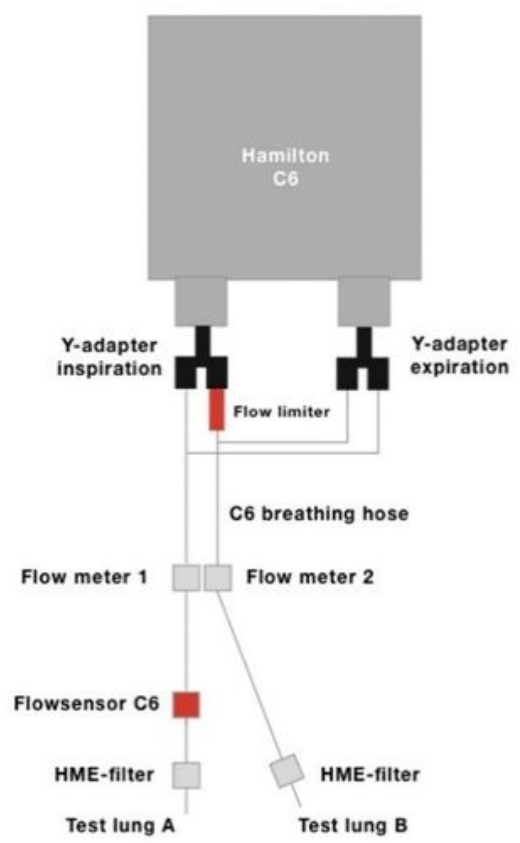

C

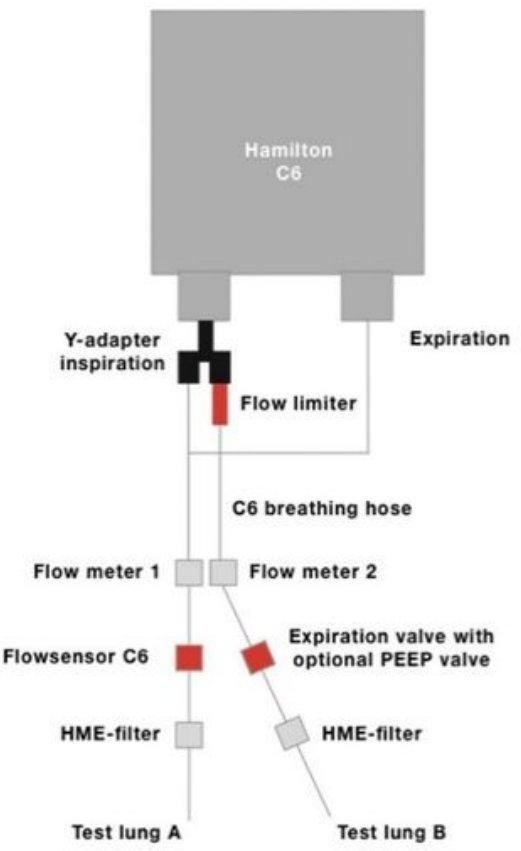

B

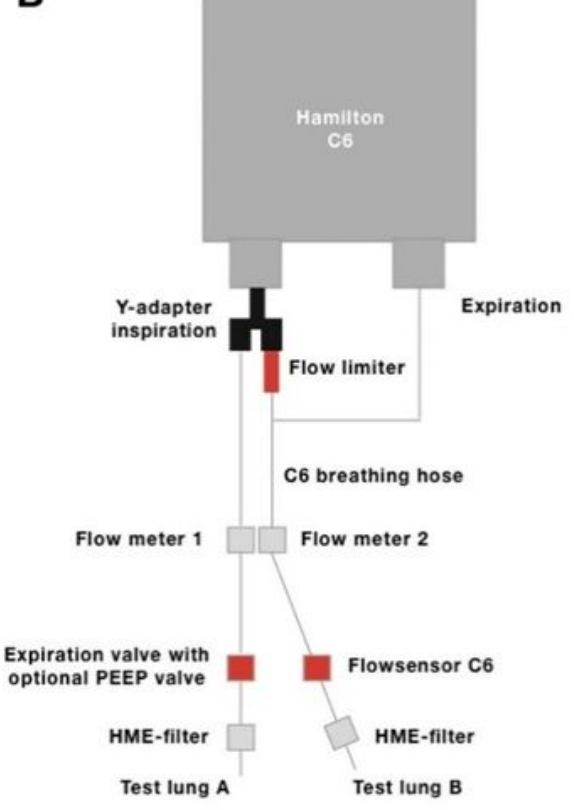

Figure 2

Illustration of a Hamilton $\mathrm{C} 6$ ventilator connected to two test lungs; Figure 2A, ventilation of two test lungs with a $y$-adapter in the in- and expiratory ventilator thigh over two double ven-tilation hoses. The 
flow limiter is placed in circuit $B$, the flow sensor in circuit $B$; Figure 2B ventilation of two lungs with only one $y$-adapter in the inspiration thigh. The flow limiter is placed in circuit B. Expiration in circuit A over a one-way expiration valve with an optional PEEP valve. The flow sensor is placed in circuit $B$ (double hose); Figure $2 \mathrm{C}$ ventilation of two lungs with $\mathrm{y}$-adapter in the inspiration thigh. Expiration in circuit $\mathrm{A}$ over a one-way expiration valve with an optional PEEP valve. The flow sensor is placed in circuit A. Circuit B double hose.

\section{Supplementary Files}

This is a list of supplementary files associated with this preprint. Click to download.

- VIDEODelayedLungVentilation1.mp4

- VIDEODelayedLungVentilation2.mp4 